\title{
Evaluation of C-reactive protein, procalcitonin, tumor necrosis factor alpha, interleukin-6, and interleukin-8 as diagnostic parameters in sepsis-related fatalities
}

\author{
Bettina Schrag • Pascale Roux-Lombard • \\ Deborah Schneiter • Paul Vaucher • Patrice Mangin • \\ Cristian Palmiere
}

Received: 15 February 2011 / Accepted: 24 June 2011 /Published online: 7 July 2011

(C) Springer-Verlag 2011

\begin{abstract}
The aims of this study were to investigate the usefulness of serum C-reactive protein, procalcitonin, tumor necrosis factor alpha, interleukin-6, and interleukin-8 as postmortem markers of sepsis and to compare C-reactive protein and procalcitonin values in serum, vitreous humor, and cerebrospinal fluid in a series of sepsis cases and control subjects, in order to determine whether these measurements may be employed for the postmortem diagnosis of sepsis. Two study groups were formed, a sepsis group (eight subjects coming from the intensive care unit of two university hospitals, with a clinical diagnosis of sepsis in vivo) and control group (ten autopsy cases admitted to two university medicolegal centers, deceased from natural and unnatural causes, without elements to
\end{abstract}

Bettina Schrag and Cristian Palmiere equally contributed as first authors to this work.

B. Schrag $\cdot$ P. Vaucher $\cdot$ P. Mangin

University Centre of Legal Medicine, Lausanne-Geneva,

Rue Michel-Servet 1,

1211 Geneva 4, Switzerland

P. Roux-Lombard

Division of Immunology and Allergy,

Department of Internal Medicine,

Geneva University Hospital and University of Geneva,

Rue Gabrielle Perret-Gentil 4,

Geneva CH-1211, Switzerland

P. Vaucher $\cdot$ P. Mangin $\cdot$ C. Palmiere $(\bowtie)$

University Centre of Legal Medicine, Lausanne-Geneva,

Rue du Bugnon 21,

1011 Lausanne, Switzerland

e-mail: cristian.palmiere@chuv.ch

D. Schneiter

University of Geneva,

Geneva, Switzerland presume an underlying sepsis as the cause of death). Serum C-reactive protein and procalcitonin concentrations were significantly different between sepsis cases and control cases, whereas serum tumor necrosis factor alpha, interleukin-6, and interleukin-8 values were not significantly different between the two groups, suggesting that measurement of interleukin-6, interleukin-8, and tumor necrosis factor alpha is non-optimal for postmortem discrimination of cases with sepsis. In the sepsis group, vitreous procalcitonin was detectable in seven out of eight cases. In the control group, vitreous procalcitonin was clearly detectable only in one case, which also showed an increase of all markers in serum and for which the cause of death was myocardial infarction associated with multiorganic failure. According to the results of this study, the determination of vitreous procalcitonin may be an alternative to the serum procalcitonin for the postmortem diagnosis of sepsis.

Keywords Sepsis · Interleukins · Procalcitonin · C-reactive protein $\cdot$ Postmortem biochemistry

\section{Introduction}

The term sepsis is used to define the systemic inflammatory response to an infectious agent (i.e., bacterial, viral, fungal, or parasitic). Despite the use of new treatment modalities, improvement in technology, and increased experience, the sepsis mortality rate remains high [1].

Sepsis can be difficult to distinguish from other noninfectious systemic inflammatory responses in critically ill patients admitted to the hospital with clinical signs of acute inflammation. Thus, there is an unmet need for 
clinical or laboratory tools distinguishing between the systemic inflammatory response syndrome and the various forms of sepsis [2].

In the forensic routine, sepsis as cause of death remains difficult to diagnose due to insufficient or absent medical information at the moment of the autopsy, contaminated blood or tissue, nonspecific autopsy findings (myocardial ischemia, pulmonary edema, hypoxic liver damage, mesenteric ischemia, gastrointestinal hemorrhages, spleen infarction, kidney ischemia, and brain edema) as well as histological observations, which may in effect have an infectious or noninfectious origin and are neither specific nor sensitive for recognizing sepsis-related fatalities [3-5].

Immunohistochemical detection of molecules of interest such as chemokine receptors, integrins, and intercellular adhesion molecules has been proposed as valuable diagnostic tools for the postmortem diagnosis of sepsis [6-8]. However, other parameters are needed to clearly establish a postmortem diagnosis of sepsis [8].

A large number of clinical studies have been performed in the last decades to correlate cytokines levels with sepsis and patient prognosis. Among the potentially useful sepsis markers, tumor necrosis factor alpha (TNF- $\alpha$ ), interleukin 2 (IL-2), interleukin 6 (IL-6), interleukin 8 (IL-8), and interleukin-10 (IL-10) have been proposed to be the most promising candidates [1, 2, 9].

Harbarth et al. investigated the diagnostic value of procalcitonin, IL-6, and IL- 8 in a group of severely ill patients admitted to hospital with signs of acute inflammation in order to assess whether these markers were helpful in distinguishing sepsis from severe systemic noninfectious inflammations. They found that procalcitonin was the best indicator of infection in newly admitted critically ill patients, whereas measurement of IL-6 and IL-8 was nonoptimal for discriminating patients with infectious from those with noninfectious diseases [2].

Balci et al. investigated the procalcitonin levels at early diagnosis and differentiation in patients with systemic inflammatory response syndrome and sepsis, in comparison with C-reactive protein (CRP), TNF- $\alpha$, IL-2, IL-6, and IL8 , in an unselected population of patients admitted to an intensive care unit and suffering from a broad range of diseases. Their results showed that procalcitonin was a more accurate marker for differentiating between patients suffering from systemic inflammatory response syndrome and those with sepsis [1].

Heper et al. evaluated serum CRP, procalcitonin, TNF- $\alpha$, and IL-10 levels within the first $72 \mathrm{~h}$ of admission to hospital in patients with community-acquired sepsis, severe sepsis, and septic shock, in order to determine the diagnostic value of these markers in differentiating sepsis from severe sepsis, especially at the early stages and establish their prognostic value in predicting the outcome at admission. They found that procalcitonin and IL-10 were more useful in discriminating between sepsis and severe sepsis, whereas TNF- $\alpha$ and IL-10 were more useful in predicting which cases were likely to have a fatal outcome [9].

In forensic practice, several authors focused on the use of serum procalcitonin, CRP, and IL-6 for the postmortem discrimination between sepsis and non-septic underlying causes of death. In this study, we investigated serum CRP, procalcitonin, TNF- $\alpha$, IL-6, and IL- 8 in a series of sepsisrelated fatalities and control subjects in order to evaluate their usefulness as postmortem markers of sepsis. We also compared CRP and procalcitonin values in serum, vitreous humor, and cerebrospinal fluid in a series of sepsis-related fatalities and control subjects in order to determine their applicability in the postmortem diagnosis of death due to sepsis.

\section{Materials and methods}

\section{Subjects}

Two study groups were formed (sepsis group and control group) according to whether there was an underlying clinical diagnosis of sepsis as the cause of death based on the medical records as well as on the autopsy findings. The sepsis group ( $n=8$, males, individual ages $4-78$ years old, mean age 46 years) consisted of eight subjects, from the intensive care unit of two university hospitals, with a welldocumented previous history and clinical diagnosis of sepsis in vivo according to the definition of the American College of Chest Physicians/Society of the Critical Care Medicine Consensus Conference [10], confirmed by postmortem examination. The period of septic condition was between 2 and 6 days. The cause of death was multiple organ failure due to sepsis in all cases. Postmortem microbiological investigations performed on cardiac blood revealed the presence of Klebsiella pneumoniae (two cases), Escherichia coli (two cases), and Staphylococcus aureus (one case). In three cases, postmortem microbiological investigation revealed no clearly identifiable pathogens.

The non-sepsis group ( $n=10$, eight males, two females, individual ages 17-69 years old, mean age 44 years) consisted of ten autopsy cases, from two university medicolegal centers, with death due to various natural (myocardial infarction and myocardial insufficiency) and unnatural (hanging, electrocution, trauma, intoxication) causes. No individuals in this study group had medical history of septic condition prior to death, and no other disease was found at the autopsy except for the cause of death (myocardial infarction $n=2$, myocardial insufficiency $n=2$, trauma $n=1$, intoxication $n=3$, electrocution $n=1$, and 
hanging $n=1)$. Cardiopulmonary resuscitation was attempted in eight cases. In none of these cases the autopsy findings gave any element suggesting an underlying infectious disease, and no postmortem microbiological investigation was carried out.

\section{Samples collection}

Serum samples

Postmortem blood samples were collected by aspiration with a sterile needle and a syringe from the femoral vein at defined time intervals between 2 and $72 \mathrm{~h}$ postmortem from the individuals included in both study groups. All blood samples were centrifuged immediately after collection at $1,500 \mathrm{rpm}$ for $15 \mathrm{~min}$. After centrifugation, the serum was frozen at $-20^{\circ} \mathrm{C}$.

Vitreous humor samples

Postmortem vitreous humor samples were collected by aspiration using a sterile needle and a syringe. Right and left vitreous humor samples were collected before autopsy through a scleral puncture at the lateral canthus and mixed together. All vitreous samples were centrifuged immediately after collection at 1,500 rpm for $15 \mathrm{~min}$. After centrifugation, the samples were frozen at $-20^{\circ} \mathrm{C}$.

\section{Cerebrospinal fluid samples}

Cerebrospinal fluid samples were collected by aspiration using a sterile needle and a syringe by suboccipital puncture. All cerebrospinal fluid samples were immediately frozen after collection at $-20^{\circ} \mathrm{C}$.

\section{Analysis}

Determination of C-reactive protein

SYNCHRON ${ }^{\circledR}$ C-Reactive Protein Reagent (Beckman Coulter, Inc., Brea, USA) was used to measure CRP concentration by a turbidimetric method according to the supplier's instructions. In the reaction, CRP combines with a specific antibody to form insoluble antigen-antibody complexes. SYNCHRON systems automatically proportion the appropriate sample and reagent volumes into a cuvette, one part sample to 26 parts reagent. The system monitors the change in absorbance at $340 \mathrm{~nm}$. This change in absorbance is proportional to the concentration of CRP in the sample and is used by the systems to calculate and express CRP concentration, based upon a multipoint, nonlinear calibration curve. Results were expressed in milligrams per liter. A value greater than $10 \mathrm{mg} / \mathrm{l}$ was considered to be elevated.

Determination of procalcitonin

KRYPTOR $^{\circledR}(B \cdot R \cdot A \cdot H \cdot M \cdot S$, Henningsdorf, Germany) was used to measure procalcitonin concentration. KRYPTOR ${ }^{\circledR}$ uses time-resolved amplified cryptate emission technology, based on a non-radiative transfer of energy. This transfer takes place between two fluorescent tracers: europium cryptate (donor) and XL665 (acceptor). The signal measured during the formation of the antigen-antibody complex is accompanied by amplification. KRYPTOR ${ }^{\circledR}$ assays are homogeneous, without separation or washing. The molecules of procalcitonin present in the assay samples are sandwiched between the antibodies. The intensity of the signal is proportional to the amount of procalcitonin. The shape of the standard curve is identical to that obtained by immunometric methods. Results were expressed in nanograms per milliliter. A value lower than $0.25 \mathrm{ng} / \mathrm{ml}$ was considered to be normal.

Determination of tumor necrosis factor alpha, interleukin-6, and interleukin-8

TNF- $\alpha$, IL-6, and IL- 8 were measured by a commercially available multiplex beads immunoassay, based on the Luminex platform (Fluorokine MAP Multiplex Human Cytokine Panel, R\&D Systems, Minneapolis, USA) according to supplier's instructions. Briefly, beads with defined spectral property were conjugated to the analyte-specific capture antibodies. Beads, samples, standards, and the controls were pipetted in a filter-bottomed 96-well plate and incubated at room temperature for $3 \mathrm{~h}$ while gently shaking. After three washes, the biotinylated detector antibody cocktail was added to the wells and incubated for $1 \mathrm{~h}$ at room temperature. Streptavidin conjugated with R-phycoerythrin was added to the wells after several washings and incubated for $30 \mathrm{~min}$. The data (mean fluorescence intensity) were then read by the Bio-Plex 200 array reader (Bio-Rad Laboratories, Hercules, CA, U.S. A.) which uses Luminex xMAPTM Technology (Luminex Corporation, Austin, TX, U.S.A.). Sample concentrations were calculated using standard curves. Results were expressed in picograms per milliliter.

Statistical method

Means, standard deviations (SD), medians, minimum and maximum values were reported for each group. Observed values were plotted, and normal distribution was assessed measuring skewness and kurtosis. As distribution was 
skewed, differences between sepsis and control group for serum procalcitonin, CRP, TNF- $\alpha$, IL- 6 , and IL- 8 were compared using nonparametrical Mann-Whitney $U$ test. The significance level was set at 0.05 . Correlations between serum, vitreous humor and cerebrospinal fluid were tested using Spearman's correlation coefficient.

\section{Results}

Individual serum procalcitonin, CRP, TNF- $\alpha$, IL-6, and IL8 values are reported in Fig. 1. Values were skewed and cannot be considered as normally distributed. Individual procalcitonin and CRP values in the vitreous humor and in the cerebrospinal fluid for sepsis cases and control cases are reported in Table 1.

Serum CRP levels were elevated in all sepsis cases, with values varying from 23 to $272 \mathrm{mg} / \mathrm{l}$, whereas serum procalcitonin levels were elevated in seven out of eight cases, with values varying from 0.03 to $218 \mathrm{ng} / \mathrm{ml}$.

Most sepsis cases also showed high values of serum TNF- $\alpha$ (six cases out of eight, with values varying from 2.92 to $249 \mathrm{pg} / \mathrm{ml}$ ), whereas serum IL-6 and IL-8 levels were elevated in all sepsis cases (with values varying from 81 to $75,296 \mathrm{pg} / \mathrm{ml}$ for IL-6 and from 79 to $3,115 \mathrm{pg} / \mathrm{ml}$ for IL-8). a) CRP

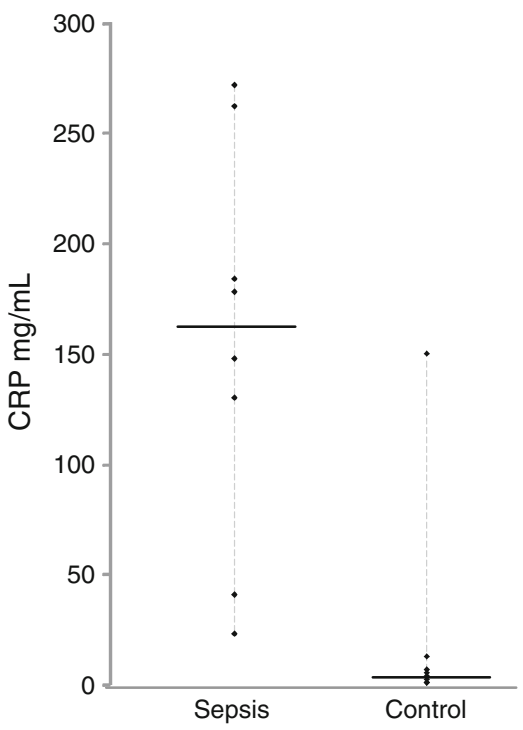

\section{d) IL-6}

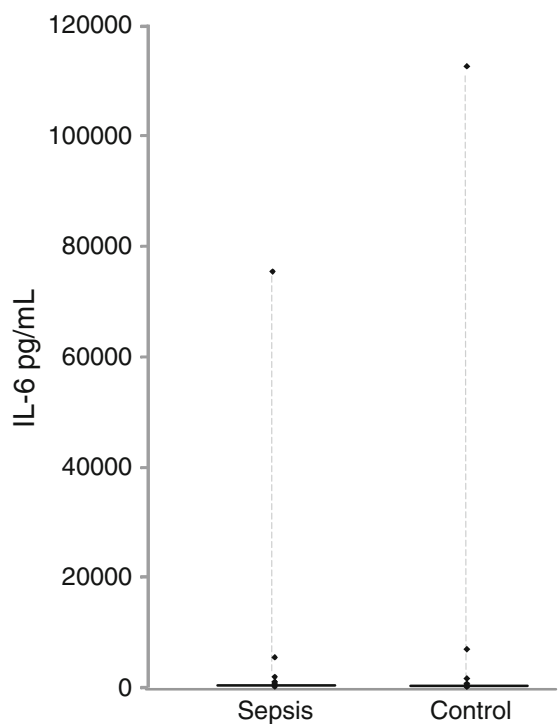

b) PCT

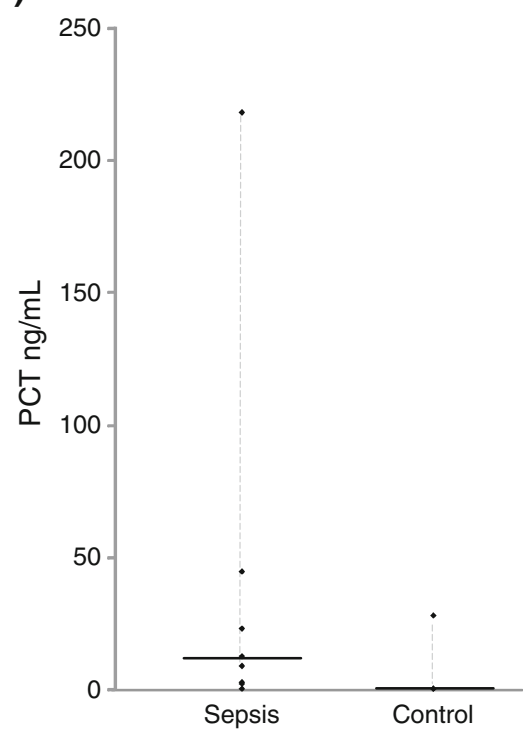

e) IL-8

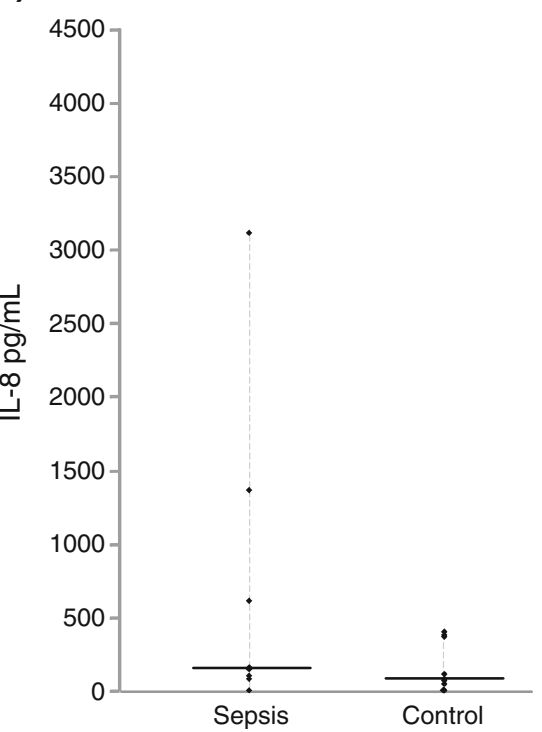

c) TNF- $\alpha$

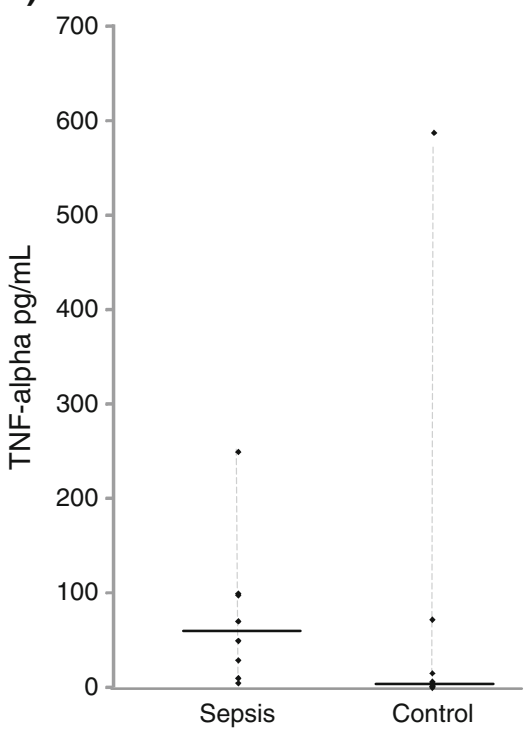

Fig. 1 Individual serum values of a CRP, b PCT, $\mathbf{c}$ TNF- $\alpha$, d IL-6, and e IL-8 
Table 1 Procalcitonin and CRP values in the vitreous humor and in the cerebrospinal fluid for sepsis cases and control cases

\begin{tabular}{|c|c|c|c|c|c|c|}
\hline \multirow[t]{2}{*}{ Group } & \multirow[t]{2}{*}{ Agent/cause of death } & \multirow[t]{2}{*}{ Age (years) } & \multicolumn{2}{|c|}{ Vitreous humor } & \multicolumn{2}{|c|}{ Cerebrospinal fluid } \\
\hline & & & CRP & PCT & CRP & PCT \\
\hline \multirow[t]{8}{*}{ Sepsis } & K. pneumoniae & 78 & 0 & 0.13 & 0 & 0.47 \\
\hline & E. coli & 28 & 1 & 0.19 & 5 & 0.22 \\
\hline & - & 38 & 0 & 0.22 & 12 & 0 \\
\hline & - & 39 & 0 & 0.85 & 2 & 4.06 \\
\hline & S. aureus & 66 & 2 & 0.68 & 0 & 0 \\
\hline & E. coli & 73 & 8 & 0.37 & 0 & 0 \\
\hline & - & 42 & 0 & 0 & 0 & 0.06 \\
\hline & K. pneumoniae & 4 & 0 & 0.84 & 1 & 24.65 \\
\hline \multirow[t]{10}{*}{ Control } & Trauma & 54 & 0 & 0 & 0 & 0.04 \\
\hline & Intoxication & 37 & 0 & 0 & 0 & 0.06 \\
\hline & Hanging & 54 & 0 & 0 & 0 & 0.07 \\
\hline & Myocardial infarction & 48 & 0 & 0 & 0 & 0.03 \\
\hline & Intoxication & 45 & 0 & 0.02 & 0 & 0.09 \\
\hline & Myocardial insufficiency & 35 & 0 & 0.04 & 0 & 0.05 \\
\hline & Intoxication & 34 & 0 & 0 & 0 & 0.1 \\
\hline & Myocardial infarction & 42 & 0 & 0 & 0 & 0.07 \\
\hline & Myocardial insufficiency & 32 & 1 & 0.34 & 4 & 4.17 \\
\hline & Electrocution & 17 & 0 & 0 & 2.2 & 0 \\
\hline
\end{tabular}

In the control group, serum procalcitonin levels were lower than $0.5 \mathrm{ng} / \mathrm{ml}$ in nine out of ten cases. In one case, the serum procalcitonin value was $27.75 \mathrm{ng} / \mathrm{ml}$. The same case showed high values of CRP $(150 \mathrm{mg} / \mathrm{l}), \mathrm{TNF}-\alpha$ $(13.86 \mathrm{pg} / \mathrm{ml})$, IL-6 $(6,798 \mathrm{pg} / \mathrm{ml})$, and IL-8 $(371 \mathrm{pg} / \mathrm{ml})$. The cause of death in this case was myocardial insufficiency associated with multi-organic failure. The autopsy findings did not give any element to suspect an underlying infectious disease, even if an infection obviously cannot be totally excluded.

Serum CRP levels in the control group were lower than $15 \mathrm{mg} / \mathrm{l}$ in nine cases out of ten (one case showed a concentration of $150 \mathrm{mg} / \mathrm{l}$, as previously reported). Serum TNF- $\alpha$ values varied in the control group from 0.1 to $586 \mathrm{pg} / \mathrm{ml}$, serum IL-6 from 16 to $112,404 \mathrm{pg} / \mathrm{ml}$, and serum IL-8 from 0 to $399 \mathrm{pg} / \mathrm{ml}$.

In the sepsis group, CRP values in the vitreous and in the cerebrospinal fluid varied from undetectable to $8 \mathrm{mg} / \mathrm{l}$ (for vitreous) and from undetectable to $12 \mathrm{mg} / \mathrm{l}$ (for cerebrospinal fluid). In the control group, CRP in the cerebrospinal fluid was not detectable in eight out of ten cases; in the vitreous humor, CRP was not detectable in nine out of ten cases.

One case showed a CRP concentration in cerebrospinal fluid of $4 \mathrm{mg} / \mathrm{l}$ and a CRP concentration in vitreous humor of $1 \mathrm{mg} / \mathrm{l}$. As expected, this case also showed high serum procalcitonin, CRP, TNF- $\alpha$, IL- 6 , and IL- 8 values.

In the sepsis group, vitreous procalcitonin was detectable in seven out of eight cases, with values varying from undetectable (one case) to $0.85 \mathrm{ng} / \mathrm{ml}$. In the cerebrospinal fluid, procalcitonin values varied from undetectable (three cases) to $24.65 \mathrm{ng} / \mathrm{ml}$.

In the control group, procalcitonin values in the cerebrospinal fluid were lower than $0.1 \mathrm{ng} / \mathrm{ml}$ in nine out of ten cases, with only one case showing a value of $4.17 \mathrm{ng} /$ $\mathrm{ml}$ (the case that showed high values of all serum markers). In the vitreous humor, procalcitonin levels were lower than $0.05 \mathrm{ng} / \mathrm{ml}$, with only one case showing a value of $0.34 \mathrm{ng} /$ $\mathrm{ml}$ (the case that showed high values of all serum markers).

We noted significant differences between sepsis group and control group for CRP $(p=0.001)$ and procalcitonin $(p=0.016)$, but not for TNF- $\alpha$, IL-6, and IL-8 (Table 2). Procalcitonin also showed interesting correlations between different biological fluids, especially for vitreous humor (Table 3).

\section{Discussion}

In this study, we investigated serum CRP, procalcitonin, TNF- $\alpha$, IL-6, and IL- 8 in a series of sepsis-related fatalities and control subjects in order to evaluate their applicability as postmortem markers of sepsis. A total of 18 cases (eight sepsis-related fatalities and ten control cases) were included in this study. According to our observations, serum CRP and procalcitonin concentrations were significantly different between sepsis cases and control subjects, whereas serum TNF- $\alpha$, IL-6, and IL-8 values were not significantly 
Table 2 Significant differences between sepsis group and control group for CRP and procalcitonin

\begin{tabular}{|c|c|c|c|c|c|}
\hline & \multicolumn{2}{|l|}{ Sepsis $(n=8)$} & \multicolumn{2}{|l|}{ Control $(n=10)$} & \multirow[t]{2}{*}{ Mann-Whitney $U$ test } \\
\hline & Mean (SD) & Median (min-max) & Mean (SD) & Median (min-max) & \\
\hline \multicolumn{6}{|l|}{ Serum } \\
\hline CRP (mg/ml) & $154.4(90.7)$ & $163(23-272)$ & $19.0(46.2)$ & $4.5(1-150)$ & $P=0.001$ \\
\hline Procalcitonin (ng/ml) & $38.9(73.8)$ & $10.7(0.03-218)$ & $2.9(8.7)$ & $0.05(0-27.7)$ & $P=0.016$ \\
\hline TNF- $\alpha(\mathrm{pg} / \mathrm{ml})$ & $75.2(79.2)$ & $58.9(2.9-249)$ & $69.5(182.7)$ & $4.0(0.08-586)$ & $P=0.076$ \\
\hline IL-6 (pg/ml) & $10,677(26,162)$ & $897(81-75,296)$ & $12,201(35,269)$ & $277(16-112,403)$ & $P=0.182$ \\
\hline IL-8 (pg/ml) & $714(1,064)$ & $151(79-3,115)$ & $157(159)$ & $76(0.01-399)$ & $P=0.051$ \\
\hline \multicolumn{6}{|l|}{ Vitreous humor } \\
\hline CRP (mg/ml) & $1.4(2.8)$ & $0(0-8)$ & $0.1(0.32)$ & $0(0-1)$ & $P=0.143$ \\
\hline Procalcitonin (ng/ml) & $0.41(0.33)$ & $0.29(0-0.9)$ & $0.04(0.11)$ & $0(0-0.34)$ & $P=0.005$ \\
\hline \multicolumn{6}{|l|}{ Cerebrospinal fluid } \\
\hline CRP (mg/ml) & $2.5(4.2)$ & $0.5(0-12)$ & $0.62(1.4)$ & $0(0-4)$ & $P=0.204$ \\
\hline Procalcitonin (ng/ml) & $3.7(8.6)$ & $0.14(0-25)$ & $0.47(1.3)$ & $0.06(0-4.2)$ & $P=0.788$ \\
\hline
\end{tabular}

different between the sepsis group and the control group. These results confirm those of the clinical studies performed by Balci et al. [1] and Harbarth et al. [2].

Multiple organ failure of various noninfectious causes (trauma, burn, myocardial infarction, pancreatitis) is well known to induce a rise in inflammatory cytokine levels. Therefore, it is not surprising that IL-6, IL-8, or TNF- $\alpha$ is not appropriate to discriminate between septic or non-septic causes of death $[5,11]$.

High serum procalcitonin concentrations were first described in children with severe bacterial infections by Assicot and co-workers, and were suggested to be a specific marker for bacterial infection. Moreover, procalcitonin concentrations seemed to bear a correlation with the severity of sepsis [12].

Tsokos et al. investigated the use of procalcitonin as a postmortem marker of sepsis and its usefulness as a biochemical parameter in the forensic elucidation of death due to sepsis. They analyzed postmortem blood concentration of procalcitonin in two study groups (a sepsis group and a control group), compared with procalcitonin concentrations measured in antemortem blood samples. Their results showed that, in the sepsis group, serum procalcitonin levels were elevated in all patients, whereas in the

Table 3 Correlation between vitreous humor and cerebrospinal fluid, $\mathrm{CRP}$, and procalcitonin values and serum values

\begin{tabular}{llll}
\hline & Vitreous humor & & Cerebrospinal fluid \\
\cline { 2 - 2 } $\begin{array}{l}\text { Spearman's correlation } \\
\text { coefficient }(p \text { value })\end{array}$ & & $\begin{array}{l}\text { Spearman's correlation } \\
\text { coefficient }(p \text { value })\end{array}$ \\
\hline CRP & $0.605(p=0.008)$ & & $0.445(p=0.064)$ \\
Procalcitonin & $0.871(p<0.0001)$ & & $0.309(p=0.213)$ \\
\hline
\end{tabular}

control group, procalcitonin levels were not detectable in most cases, suggesting that procalcitonin could be considered a suitable biochemical postmortem marker of sepsis. According to the authors, measurements of procalcitonin levels in sepsis-related fatalities seemed to be reasonable until at least approximately $140 \mathrm{~h}$ after death. The authors also emphasized that, compared to other potential biochemical postmortem markers of sepsis (TNF- $\alpha$, IL-6, and CRP), procalcitonin seemed to be more stable even after repeated freezing and thawing of the samples [4].

In the last years, several studies have been performed in order to demonstrate the usefulness in forensic practice of CRP and IL-6 in sepsis-related fatalities. All studies agreed to consider high postmortem levels of these markers to be a nonspecific indicator of an underlying inflammatory process rather than a specific marker of sepsis.

Uhlin-Hansen investigated the CRP levels in postmortem blood compared with the CRP levels measured within the last $24 \mathrm{~h}$ prior to death. The results of this study showed that postmortem CRP values in average were reduced by $35 \%$ compared to antemortem values. However, high CRP levels in postmortem blood seemed to be a good marker for ongoing inflammatory processes prior to death [13].

Tsokos et al. investigated the serum CRP and IL-6 levels in sepsis-related fatalities and in a control group during the early postmortem period, compared with CRP and IL-6 levels measured in antemortem blood samples. They found that, in the sepsis-related fatalities, antemortem and postmortem CRP and IL-6 levels were significantly elevated in all patients, whereas in the control cases, CRP and IL-6 levels measured in postmortem blood showed slight increases, this being in only a limited number of cases. The authors specified that several clinical conditions associated with high IL-6 and/or CRP levels as a result of 
the systemic inflammatory response syndrome (for example, traumas or burn injuries) should be taken into consideration when assessing postmortem IL-6 and CRP concentrations as biochemical postmortem markers of sepsis [5].

Fujita et al. investigated postmortem serum CRP levels in forensic autopsy cases of acute and non-acute death, with special reference to the survival time and the cause of death. They concluded that postmortem serum CRP level can be used in forensic diagnosis in a similar manner as in clinical practice [14].

Maeda et al. analyzed serum levels of CRP in forensic autopsy cases with special reference to the cause of death. They found that serum CRP was markedly higher in cases of pneumonia and chronic renal failure, mildly to moderately elevated in cases of gastrointestinal bleeding and hypothermia, and mildly elevated in some cases of sudden cardiac death and cerebrovascular stroke. The authors concluded that elevated serum CRP levels in cases of chronic renal failure may be related to the characteristics of diseases and/or infectious complications. For pneumonia, a markedly high serum CRP level may indicate the severity of infection, whereas a low CRP value in some infantile and elderly cases of pneumonia may be ascribed to an agedependent low inflammatory response [15].

Finally, Ishikawa et al. investigated postmortem CRP levels in forensic autopsy cases, with special regard to systemic inflammatory response syndrome and infectious diseases. They observed markedly elevated serum CRP levels in cases of delayed death due to trauma, involving systemic inflammatory response syndrome. Markedly elevated serum CRP levels were also observed in cases of fatal bacterial infections [16].

In the present study, we also compared CRP and procalcitonin values in serum, vitreous humor, and cerebrospinal fluid in a series of sepsis-related fatalities and control subjects. We did so in order to determine their applicability in the postmortem diagnosis of death due to sepsis. According to our results, procalcitonin values in vitreous humor are statistically different between the sepsis group and the control group (Table 2). Moreover, a significant linear correlation was observed between CRP values in serum and in cerebrospinal fluid, as well as between procalcitonin values in serum and in vitreous humor (Table 3). Hence, even if further studies are needed to confirm these results, these first data suggest that the measurement of procalcitonin in vitreous humor could be an alternative to serum in cases where serum is not available.

This study represents a first analysis of CRP and procalcitonin in serum, vitreous humor, and cerebrospinal fluid in a series of sepsis-related fatalities and control subjects. It aims to evaluate their applicability as biochemical parameters of sepsis.
Astrup and Thomsen investigated postmortem blood and liver CRP levels in a series of 50 forensic autopsy cases. Their results showed that in cases when blood is not available and in cases of mild to moderate putrefaction, where bacteriological diagnosis is otherwise impossible, the determination of liver CRP can be useful in the diagnosis of severe infection [17].

In conclusion, the results of the present study confirm that procalcitonin is a more reliable parameter than other measures in the diagnosis of sepsis, thus allowing a better differentiation between sepsis-related fatalities and nonsepsis-related fatalities. Moreover, the determination of vitreous procalcitonin could be an alternative to the serum procalcitonin for the postmortem diagnosis of sepsis. Even if further studies and investigations are required to confirm these results, the data seem to indicate that other biological fluids may be collected and analyzed in order to make a postmortem diagnosis of sepsis and that vitreous procalcitonin might be considered a reliable alternative to the serum procalcitonin.

Conflict of interest The authors declare that they have no conflict of interest.

\section{References}

1. Balci C, Sungurtekin H, Gürses E, Sungurtekin U, Kaptanoglu B (2003) Usefulness of procalcitonin for diagnosis of sepsis in the intensive care unit. Crit Care 7(1):85-90

2. Harbarth S, Holeckova K, Froidevaux C, Pittet D, Ricou B, Grau GE, Vadas L, Pugin J, Network GS (2001) Diagnostic value of procalcitonin, interleukin-6, and interleukin- 8 in critically ill patients admitted with suspected sepsis. Am J Respir Crit Care Med 164(3):396-402

3. Torgersen C, Moser P, Luckner G, Mayr V, Jochberger S, Hasibeder WR, Dünser MW (2009) Macroscopic postmortem findings in 235 surgical intensive care patients with sepsis. Anesth Analg 108(6): 1841-1847

4. Tsokos M, Reichelt U, Nierhaus A, Püschel K (2001) Serum procalcitonin (PCT): a valuable biochemical parameter for the postmortem diagnosis of sepsis. Int J Legal Med 114(4-5):237243

5. Tsokos M, Reichelt U, Jung R, Nierhaus A, Püschel K (2001) Interleukin-6 and C-reactive protein serum levels in sepsis-related fatalities during the early postmortem period. Forensic Sci Int 119 (1):47-56

6. Tsokos M, Fehlauer F, Püschel K (2000) Immunohistochemical expression of E-selectin in sepsis-induced lung injury. Int J Legal Med 113(6):338-342

7. Tsokos M, Fehlauer F (2001) Postmortem markers of sepsis: an immunohistochemical study using VLA-4 (CD49d/CD29) and ICAM-1 (CD54) for the detection of sepsis-induced lung injury. Int J Legal Med 114:291-294

8. An JL, Ishida Y, Kimura A, Tsokos M, Kondo T (2009) Immunohistochemical detection of CCR2 and CX3CR1 in sepsis-induced lung injury. Forensic Sci Int 192(1-3):21-25

9. Heper Y, Akalin EH, Mistik R, Akgöz S, Töre O, Göral G, Oral B, Budak F, Helvaci S (2006) Evaluation of serum C-reactive protein, 
procalcitonin, tumor necrosis factor alpha, and interleukin-10 levels as diagnostic and prognostic parameters in patients with community-acquired sepsis, severe sepsis, and septic shock. Eur J Clin Microbiol Infect Dis 25(8):481-491

10. American College of Chest Physicians, Society of Critical Care Medicine Consensus Conference Committee (1992) Definition for sepsis and organ failure and guidelines for the use of innovative therapies in sepsis. Crit Care Med 20:864-874

11. Keel M, Trentz O (2005) Pathophysiology of polytrauma. Injury 36(6):691-709

12. Assicot M, Gendrel D, Carsin H, Raymond J, Guilbaud J, Bohuon C (1993) High serum procalcitonin concentrations in patients with sepsis and infection. Lancet 341(8844):515-518

13. Uhlin-Hansen L (2001) C-reactive protein (CRP), a comparison of pre- and postmortem blood levels. Forensic Sci Int 124(1):32-35
14. Fujita MQ, Zhu BL, Ishida K, Quan L, Oritani S, Maeda H (2002) Serum C-reactive protein levels in postmortem blood - an analysis with special reference to the cause of death and survival time. Forensic Sci Int 130(2-3):160-166

15. Maeda H, Zhu BL, Bessho Y, Ishikawa T, Quan L, Michiue T, Zhao D, Li DR, Komatsu A (2008) Postmortem serum nitrogen compounds and C-reactive protein levels with special regard to investigation of fatal hyperthermia. Forensic Sci Med Pathol 4 (3): $175-180$

16. Ishikawa T, Hamel M, Zhu BL, Li DR, Zhao D, Michiue T, Maeda $\mathrm{H}$ (2008) Comparative evaluation of postmortem serum concentrations of neopterin and C-reactive protein. Forensic Sci Int 179 (2-3): 135-143

17. Astrup BS, Thomsen JL (2007) The routine use of C-reactive protein in forensic investigations. Forensic Sci Int 172(1):49-55 